\title{
Atypical femoral neck fracture after prolonged bisphosphonate therapy
}

\author{
Kwang-kyoun Kim ${ }^{1}$, Young-wook Park², Tae-hyeong Kim ${ }^{1}$, Kyung-deok Seo ${ }^{1}$ \\ Departments of ${ }^{1}$ Orthopaedic Surgery and ${ }^{2}$ Pathology, Konyang Unversity Hospital, Daejeon, Korea
}

Of the drugs developed to prevent and treat osteoporosis, bisphosphonate has played a very important role in preventing osteoporotic fractures. However, case reports describing atypical femoral fractures in patients using long-term bisphosphonates have emerged. The majority of atypical femur fractures occurs in the lateral aspect of the subtrochanteric or femur diaphysis, which is explained by accumulation of tensile stress in these areas. Although the superior cortex of the femur neck withstands maximum tensile stress, to our knowledge, there have been only two reports (three cases) of atypical femoral neck fracture. In addition, none of those case reports revealed detailed pathology related to suppressed bone turnover rate. We encountered an incomplete femoral neck fracture and diagnosed it as "atypical" on the basis of the patient's lack of trauma and medication history and pathological findings. For patients with groin pain, minimal or no trauma, and a history of long-term bisphosphonate use, an atypical femoral neck fracture should be considered.

Key Words: Atypical femur fracture; Femur neck fracture; Tensile strength; Bisphosphonate

Received: April 7, 2020 Revised: May 4, 2020 Accepted: May 14, 2020

Corresponding Author: Kwang-Kyoun Kim, MD, Department of Orthopaedic Surgery, 158 Gwanjeodong-ro, Seo-gu, Daejeon 35365, Korea

Tel: +82-42-600-6902, Fax: +82-42-600-9964, E-mail: kimajouos@gmail.com

Bisphosphonates reduce the overall risk of osteoporotic fractures and have a long-term beneficial effect. However, they reduce bone turnover and allow micro-cracks to accumulate over time, leading to atypical femoral fracture (AFF) [1]. There have been many reports of AFF in the subtrochanter or the diaphysis that were attributed to accumulation of tensile stress in these regions [2]. According to Koch [3], the greatest tensile strength occurs at the superior cortex of the femoral neck. However, to our knowledge, there have been only three cases of atypical femoral neck fracture (AFNF) [4,5]. In addition, none of those case reports revealed evidence related to suppressed bone turnover rate. Here, we report a case of AFNF with detailed pathological findings and a literature review.

\section{CASE REPORT}

A 71-year-old female was referred to our clinic with a 3-month history of pain in the right groin. Her pain was not preceded by a fall or any injury. Three weeks before presenting to our clinic, the pain worsened so that she could not bear weight on the right hip, and she underwent hip radiography and magnetic resonance imaging (MRI) at the referring hospital, where she was conservatively treated for a bone contusion. The patient had been taking $3 \mathrm{mg}$ of intravenous ibandronate sodium every 3 months over the previous 4 years for osteoporosis. At admission, the bone mineral densities (T-score) of the lumbar spine, total hip, and femoral neck were $-3.6,-1.4$, and -1.2 , respectively. On physical examination, there was pain limitation to range of motion and a positive log roll test for the right hip.

Laboratory tests showed normal adjusted serum calcium (8.9 $\mathrm{mg} / \mathrm{dL}$; normal range, 8.0 to 10.5 ), inorganic phosphorus (3.2 $\mathrm{mg} / \mathrm{dL}$; normal range, 2.5 to 4.5$)$, serum creatinine $(0.7 \mathrm{mg} / \mathrm{dL}$; normal range, 0.6 to 1.1 ), and alkaline phosphatase ( $85 \mathrm{IU} / \mathrm{L}$; normal range, 40 to 120). However, 25-hydroxycholecalciferol $(17.8 \mathrm{ng} / \mathrm{mL}$; normal range, 30 to 80 ) and urinary excretion of the $\mathrm{N}$-telopeptide of type I collagen ( $8 \mathrm{nM}$ bone collagen equivalents/nM creatinine; normal range, 11 to 91) were decreased.

Hip radiograph performed at our hospital upon admission showed a more definite and extended incomplete right femoral neck fracture (Fig. 1A, B), whereas a 1.5T MRI conducted at the referral hospital had shown a decreased signal intensity without fracture on the right femur neck (Fig. 1C).

A specimen that included the fracture line was obtained and stained with hematoxylin and eosin (H\&E) (Fig. 2A). The frac- 

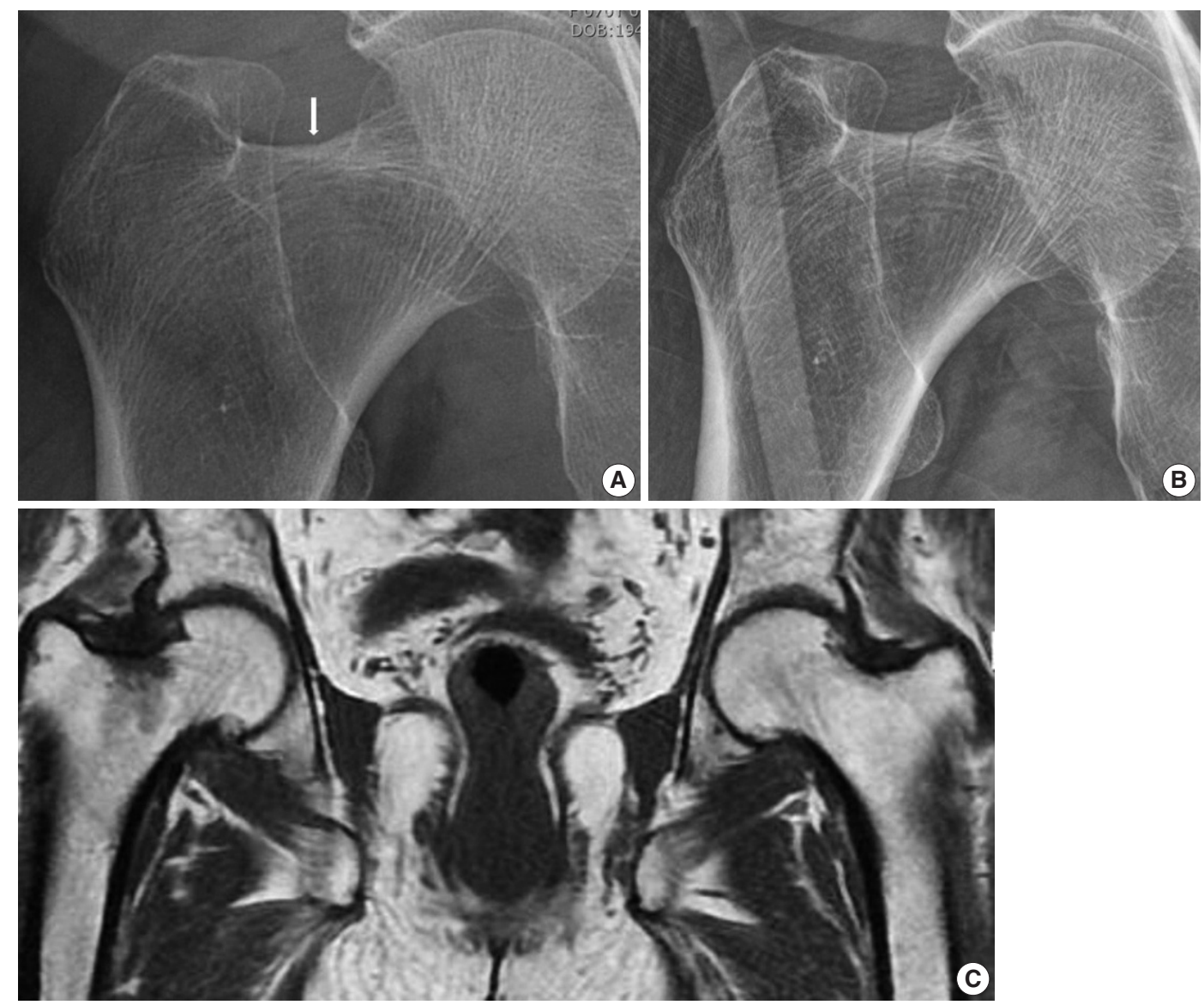

Fig. 1. (A) A radiograph performed 3 weeks before referral to our hospital shows a minimal fracture line (white arrow) on the superior cortex of the right femoral neck. (B) A radiograph performed at our hospital at admission shows a more definite and nearly vertical radiolucent line (fracture) in the right femoral neck. (C) T1 magnetic resonance imaging shows a decreased signal intensity in the superior and central aspect of the right femoral neck, which represents bone marrow edema.

ture gap appeared as a vertical, nearly empty crack, $\leq 0.1 \mathrm{~mm}$ wide, and containing mainly amorphous acellular material. No hematoma, vessels, chondrocytes, or inflammatory cells were seen within the fracture gap (Fig. 2B). The deep portion of the fracture was mostly replaced by fibrovascular tissue with a focal area of osteoid formation, indicating fracture healing (Fig. 2C). Increased remodeling activity (active osteoblastic rimming and osteoclast proliferation) was seen around the fracture gap (Fig. 2D). Osteoclasts were widely distributed, except within the fracture gap (Fig. 2). There were giant multinucleated osteoclasts, with more than 8 detached from the shallow resorption cavity (Fig. 3). The patient was diagnosed with AFNF based on history of long-term bisphosphonate therapy, suppressed bone turnover marker (urinary excretion of the $\mathrm{N}$-telopeptide of type I collagen), and radiologic and pathological findings.

Because the patient required early weight bearing, bipolar arthroplasty was performed rather than internal fixation. Bisphos- phonate treatment was discontinued, oral supplemental vitamin D was prescribed, and teriparatide was administered subcutaneously to prevent osteoporotic fracture. The patient was able to bear full weight immediately after the operation, and no pain was felt in either hip at 3 months postoperatively.

\section{DISCUSSION}

To our knowledge, only three cases of atypical femoral neck fractures have been previously reported with little evidence. Khan et al. [5] reported the pathological findings of disconnected, mainly thin trabecular bone with necrosis, hemorrhaging, and acute inflammatory cells. However, these pathological findings are not specific to AFF and are considered evidence of acute fracture in osteoporotic patients [6,7]. However, the pathology from the present case showed that the fracture gap contained amorphous acellular material and no living cells. Con- 

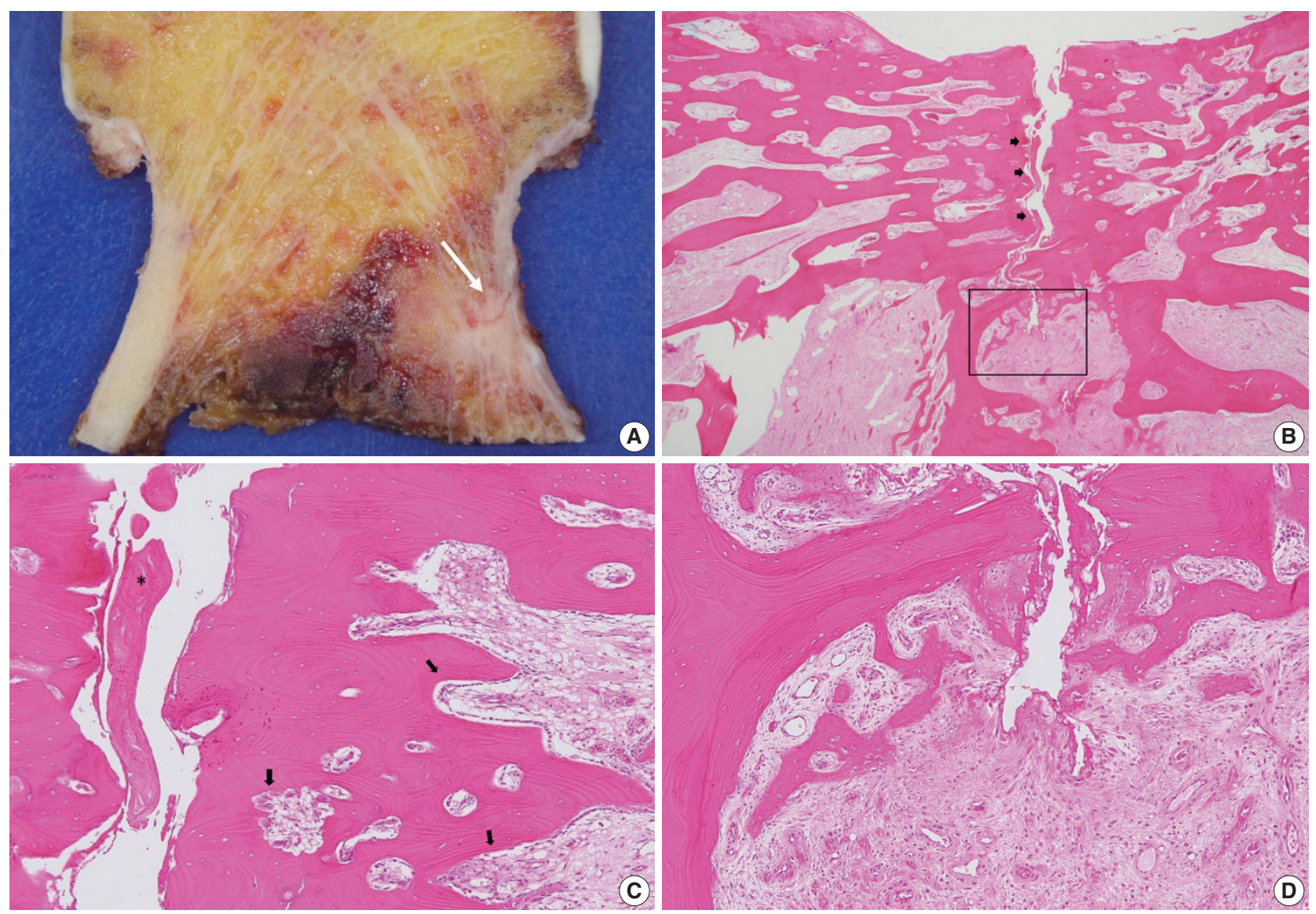

Fig. 2. (A) Specimen shows a definite incomplete fracture of the superior femoral neck (arrow). (B) A thin fracture line (arrows) extends through the entire thickness of the cortex. (C) A thin fracture gap contained amorphous acellular material (asterisk) but no hematoma, vessels, chondrocytes, or inflammatory cells. However, remodeling cavities with increased cellular activity are seen in the bone adjacent to the fracture gap (arrows). (D) A deep portion of the fracture site is mostly replaced by fibrovascular tissue with a focal area of osteoid formation representing the fracture-healing process (magnification of square area of B).

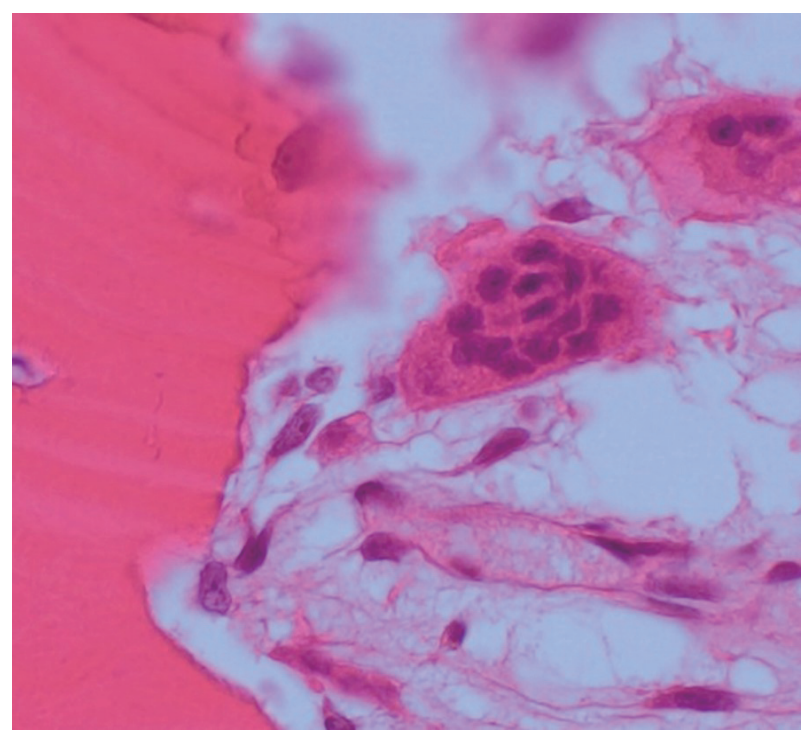

Fig. 3. A giant multinucleated osteoclast with more than 8 nuclei detached from the shallow resorption cavity. versely, the adjacent bone revealed living cells, including active osteoclasts. These findings show that the present case was not an acute fracture, but a stress fracture.

Osteoclasts normally contain up to eight nuclei before apoptosis, but in the present case, there were giant multinucleated osteoclasts with more than 8 nuclei detached from the shallow or absent resorption cavity. This is as a characteristic finding of bisphosphonate-related atypical fractures [8-10]. Osteoclast apoptosis is likely due to exposure to a high extracellular concentration of calcium released during bone absorption $[11,12]$. Bisphosphonate inhibits formation of the guanosine triphosphate-binding protein necessary for osteoclasts to form the ruffled border necessary for adhesion and resorption on bone surface; therefore, calcium concentration in the resorption cavity does not increase due to loss of absorption from long-term bisphosphonate use [13]. As a result, by reducing the signal (high extracellular calcium concentration), which is important 
for osteoclast apoptosis, the lifespan of the osteoclast increases; therefore, the number of osteoclasts does not decrease, and fusion continues to form giant multinuclear cells $[8,14]$. Giant multinuclear cells are also found in Paget's disease, secondary hyperparathyroidism, giant cell tumor, and fibrodysplasia. However, these conditions can be pathologically differentiated by number, size, and apoptosis of osteoclasts; amount of osteoids; and condition of the hematopoietic bone marrow.

Fractures originating in the femoral neck and in the intertrochanteric femur are excluded from the case definition of AFF because they are more likely to be associated with other causes such as trauma or osteoporosis. However, given that atypical fractures are clustered at the region of maximal tensile loading, the superior cortex of the femoral neck withstands maximum tensile stress, putting it at risk of atypical fractures [2,3]. We assumed that the paucity of reports on AFNFs was related to the fractures being mistaken for the transverse type of osteoporotic insufficiency fractures [15]. In osteoporosis, the pathology shows thin trabeculae disconnected from each other, enlarged areolar tissue, and increased resorption cavity, and the increased bone turnover marker is shown in laboratory testing. In the present case, urinary excretion of the N-telopeptide of type I collagen was reduced, reflecting a decrease in bone remodeling due to bisphosphonate [16,17]. Recently, atypical fractures were also reported in the ulna, tibia, ilium, and pubis [18-20]. Therefore, I propose that the case definition of AFF be limited to femoral subtrochanter and shaft that require supplementation.

There is a limitation in this study. Histomorphometry of giant multinuclear osteoclasts (i.e., scale, shape, and position) and number of giant multinuclear osteoclasts were not detailed, as only H\&E staining was performed.

In conclusion, the patient in the present case had no trauma history, long-term bisphosphonate use, reduced urinary excretion of the N-telopeptide of type I collagen, and a pathological finding of bisphosphonate-related atypical fracture comprised of amorphous acellular material and no living cells in the fracture gap and giant multinucleated osteoclast detached from the bone perimeter. For patients with groin pain with minimal or no trauma and a history of prolonged bisphosphonate use, the possibility of AFNF should be considered.

\section{Ethics Statement}

The Institutional Review Board of Konyang University Hospital (KYUH 2019-10-014) approved this study, and we received informed consent from the patient.

\section{ORCID}

Kwang-kyoun Kim https://orcid.org/0000-0002-6844-5431

Young-wook Park https://orcid.org/0000-0002-7976-6644

Tae-hyeong Kim https://orcid.org/0000-0001-7410-8813

Kyung-deok Seo https://orcid.org/0000-0001-7227-0710

\section{Author Contributions}

Conceptualization: KKK. Data curation: KKK, KDS. Investigation: KKK, YWP. Methodology: YWP, KKK. Project administration: KKK. Resources: KKK. Software: KKK. Supervision: KKK. Validation: KKK, THK. Visualization: YWP, KKK, THK. Writing-original draft: KKK. Writing-review \& editing: KKK. Approval of final manuscript: all authors.

\section{Conflicts of Interest}

The authors declare that they have no potential conflicts of interest.

\section{Funding Statement}

No funding to declare.

\section{Acknowledgments}

Thanks to Change-jin Kim and Pan-jo Kim, who assisted me in finding giant multi-nucleated cells in a cold pathology room.

\section{References}

1. Shane E, Burr D, Ebeling PR, et al. Atypical subtrochanteric and diaphyseal femoral fractures: report of a task force of the American Society for Bone and Mineral Research. J Bone Miner Res 2010; 25 : 2267-94.

2. Koh JS, Goh SK, Png MA, Ng AC, Howe TS. Distribution of atypical fractures and cortical stress lesions in the femur: implications on pathophysiology. Singapore Med J 2011; 52: 77-80.

3. Koch JC. The laws of bone architecture. Am J Anat 1917; 21: 177298.

4. Kim DH, Lee EC, Kang SK. Insufficiency fracture of ipsilateral femur neck in patient treated with long term bisphosphonate treatment: a case report. J Bone Metab 2012; 19: 159-62.

5. Khan SK, Savaridas T, Hemers JS, Maarouf Z, Orgee JM, Orr MM. Atraumatic intracapsular neck of femur fractures after prolonged bisphosphonate treatment: a new atypical variant? Clin Cases Miner Bone Metab 2016; 13: 38-41.

6. Schilcher J, Sandberg O, Isaksson H, Aspenberg P. Histology of 8 atypical femoral fractures: remodeling but no healing. Acta Orthop 2014; 85: 280-6.

7. Kajino Y, Kabata T, Watanabe K, Tsuchiya H. Histological finding of atypical subtrochanteric fracture after long-term alendronate therapy. J Orthop Sci 2012; 17: 313-8.

8. Weinstein RS, Roberson PK, Manolagas SC. Giant osteoclast formation and long-term oral bisphosphonate therapy. N Engl J Med 2009; 360: 53-62.

9. Whyte MP, Wenkert D, Clements KL, McAlister WH, Mumm S. Bisphosphonate-induced osteopetrosis. N Engl J Med 2003; 349: 457-63.

10. Jain N, Weinstein RS. Giant osteoclasts after long-term bisphosphonate therapy: diagnostic challenges. Nat Rev Rheumatol 2009; 5: 341-6.

11. Lorget F, Kamel S, Mentaverri R, et al. High extracellular calcium concentrations directly stimulate osteoclast apoptosis. Biochem Biophys Res Commun 2000; 268: 899-903. 
12. Nielsen RH, Karsdal MA, Sorensen MG, Dziegiel MH, Henriksen K. Dissolution of the inorganic phase of bone leading to release of calcium regulates osteoclast survival. Biochem Biophys Res Commun 2007; 360: 834-9.

13. Rogers MJ, Crockett JC, Coxon FP, Mönkkönen J. Biochemical and molecular mechanisms of action of bisphosphonates. Bone 2011; 49: 34-41.

14. Cremers S, Papapoulos S. Pharmacology of bisphosphonates. Bone 2011; 49: 42-9.

15. Parks TG, Baird DS, Wilson RI. Stress fractures of the neck of the femur. Ulster Med J 1970; 39: 111-8.

16. Bone HG, Hosking D, Devogelaer JP, et al. Ten years' experience with alendronate for osteoporosis in postmenopausal women. $\mathrm{N}$
Engl J Med 2004; 350: 1189-99.

17. Greenblatt MB, Tsai JN, Wein MN. Bone turnover markers in the diagnosis and monitoring of metabolic bone disease. Clin Chem 2017; 63: 464-74.

18. Osada R, Zukawa M, Kimura T. Atypical ulnar fracture associated with long-term bisphosphonate use. J Orthop Sci 2015; 20: 1132-5.

19. Breglia MD, Carter JD. Atypical insufficiency fracture of the tibia associated with long-term bisphosphonate therapy. J Clin Rheumatol 2010; 16: 76-8.

20. Patel V, Graves L, Lukert B. Pelvic fractures associated with longterm bisphosphonate therapy-case report. J Musculoskelet Neuronal Interact 2013; 13: 251-4. 